\title{
Mycobacterium fortuitum
}

National Cancer Institute

\section{Source}

National Cancer Institute. Mycobacterium fortuitum. NCI Thesaurus. Code C86538.

A species of aerobic, Gram positive, rod shaped bacterium assigned to the phylum Actinobacteria. This species is acid fast, nonmotile, urease negative and catalase, nitrate reductase, and esculin positive. M. fortuitum is found in natural and processed water sources, sewage and dirt, and can cause various clinical syndromes, including local disease after injury or surgical trauma, and disseminated infection in immunocompromised patients. 